Voix et Images

voixetimages

\title{
Des carnassiers au buffet chinois
}

\section{Ching Selao}

Volume 41, numéro 3 (123), printemps-été 2016

URI : https://id.erudit.org/iderudit/1038170ar

DOI : https://doi.org/10.7202/1038170ar

Aller au sommaire du numéro

Éditeur(s)

Université du Québec à Montréal

ISSN

0318-9201 (imprimé)

1705-933X (numérique)

Découvrir la revue

Citer ce compte rendu

Selao, C. (2016). Compte rendu de [Des carnassiers au buffet chinois]. Voix et Images, 41(3), 187-194. https://doi.org/10.7202/1038170ar d'utilisation que vous pouvez consulter en ligne.

https://apropos.erudit.org/fr/usagers/politique-dutilisation/ 


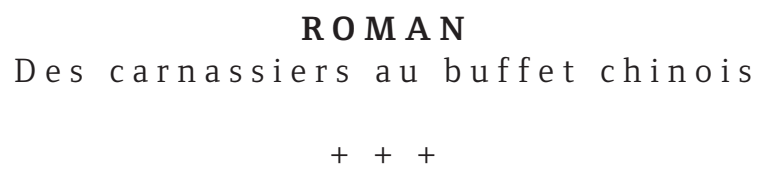

CHING SELAO

Université du Vermont (États-Unis)

Si l'œuvre de Marie-Célie Agnant connaît aujourd'hui une réception et un lectorat assez importants, elle s'est imposée graduellement, grâce à la ténacité et au travail assidu de l'auteure, qui n'a pas toujours été prise au sérieux. Mère de trois enfants, femme qui assume son féminisme et sa condition d'immigrante, bien qu'elle vive au Québec depuis plus de quarante-cinq ans, Agnant n'hésite pas, quand il le faut, à dénoncer le racisme de la société québécoise ou le sexisme de la communauté littéraire haïtienne. En tant qu'auteure qui évolue dans le milieu montréalais dominé par les hommes et connu pour faire preuve de solidarité et de soutien, elle a souvent évoqué l'attitude condescendante, voire méprisante de certains écrivains à son égard, comme l'illustre cette anecdote qu'elle a déjà racontée: «La dame qui m'avait invitée [au Salon du livre à Paris] m'a dit: "Il y a quelque chose que je ne comprends pas. Je parlais de vous à un de vos collègues, un compatriote haïtien, et il m'a répondu 'Je vous parle d'auteurs, vous me parlez de Marie-Célie Agnant'" ${ }^{\prime 1}$.» L'écrivaine ne s'est pas laissé décourager par ce genre de remarques désobligeantes et a construit, au fil des années, une œuvre composée de romans, de recueils de poésie et de livres jeunesse. Son plus récent roman, Femmes au temps des carnassiers ${ }^{2}$, aborde de nouveau les thèmes de la violence, du silence, de la mémoire et de la vengeance dans le contexte des crimes commis sous la dictature de Duvalier, problématiques qui étaient aussi au cœur d'Un alligator nommé Rosa ${ }^{3}$. Cette fameuse Rosalie Bosquet, alias Madame Max Adolphe, est d'ailleurs mentionnée dans le dernier roman (75 et 143), mais il ne s'agit plus ici de mettre en lumière le rôle des femmes tortionnaires. Femmes au temps des carnassiers offre plutôt la vision des combattantes - de l'une d'entre elles en particulier - qui ont résisté, résolues à "vivre dans la dignité, [à] vivre en femme[s] debout» (84), à une époque et dans un contexte où il aurait été si facile de fléchir tant la barbarie a atteint son paroxysme.

Le roman est inspiré d'un épisode de la vie d'Yvonne Hakim Rimpel, journaliste haïtienne et militante féministe. Dédié à sa mémoire, il met en exergue cette

1 Colette Boucher, "Québec-Haiti. Littérature transculturelle et souffle d'oralitê: une entrevue avec MarieCélie Agnant», Ethnologies, vol. 27, n 1, 2005, p. 205.

2 Marie-Célie Agnant, Femmes au temps des carnassiers, Montréal, Les Éditions du remue-ménage, 2015, 224 p.

3 Marie-Célie Agnant, Un alligator nommé Rosa, Montréal, Les Éditions du remue-ménage, 2007, 240 p. 
phrase qui résume le projet d'écriture et l'importance de faire connaître le drame qui a brutalement réduit au silence cette femme courageuse: «Cet ouvrage, simplement pour dire qu'une histoire tue est une histoire tuée.» La narration de la première partie est portée par Mika Pelrin (on remarquera les anagrammes formées à partir du nom de la vraie journaliste), et l'action se passe à Port-au-Prince, en 1958, sous le règne de Papa Doc. Quant à la seconde partie, c'est Junon, petite-fille de Mika et fille de Soledad, qui en est la narratrice, et le récit a principalement lieu à Grenade, en Espagne, de 1974 jusqu'en 1986, année où Baby Doc a fui Haïti pour se rendre «dans le sud, en villégiature, au pays des Droits de l'Homme!» (166) C'est également l'année où Junon décide d'aller en Haïti pour la première fois, afin de mieux comprendre ce qui s'est passé le 5 janvier 1958, cette terrible nuit où sa mère et sa grandmère ont été torturées et violées par des "carnassiers ». Il faut le dire, la lecture de ce roman n'est pas toujours agréable ni facile, ce qui ne l'empêche pas d'être essentielle. Ce qui la rend par ailleurs soutenable, c'est qu'Agnant décrit avec passion, voire avec violence, la solitude, la colère, l'impuissance confrontée au désir de résistance des personnages, mais nous épargne les descriptions détaillées d'actes épouvantables commis par les Tontons Macoutes. Femmes au temps des carnassiers propose notamment une réflexion à la fois sur la nécessité et la difficulté de témoigner, de mettre en mots l'horreur, ce qui constitue en quelque sorte l'héritage de Junon:

\footnotetext{
J'avais péniblement débuté des études en cinéma. Le témoignage, seul, me persuadais-je, pouvait rendre un sens à mon existence. Il me fallait donner à voir, à sentir, faire comprendre la vie des êtres humains dans l'enfer des dictatures. Je ne voulais pas inventer des vies, simplement les présenter ainsi qu'elles étaient. Comment dire l'indicible, où puiser les mots? (162)
}

Pourtant, il faut bien arriver à exprimer l'indicible, que ce soit par le biais du documentaire ou du travail journalistique, ou même à travers le cri, comme le fait Jeanne, qui a sombré dans la folie depuis la disparition de son petit-fils, puisque «[le] silence est [...] beaucoup plus violent que la violence subie» (95).

Or, si les mots permettent de donner "une forme un peu plus humaine» (159) à la douleur, de s'accrocher à un bout d'humanité, toute parole n'est pas valorisée dans ce roman. Se glisse en effet dans les confidences de Mika une critique des thérapies vers lesquelles les victimes sont poussées après le traumatisme: «Qu'y a-t-il de plus triste que le silence? je me répète sans cesse. Mais ne nous faut-il pas également résister à la fascination des confessions forcées ou impudiques, ces impulsions qui nous poussent à parler, à nous écouter parler, au risque de banaliser les choses les plus importantes?» (150) Mika est aussi celle qui refuse de se complaire dans le rôle de victime, comme elle le précise à Junon: «J'ai certes été victime d'un crime abominable et les auteurs de ce crime n'ont jamais été inquiétés. Mais j'ai toujours refusé d'adopter un discours ou une posture de victime, qui charrient souvent des relents de perversité.» (146) Avant cet événement, elle manifestait souvent ses désaccords avec sa sœur Clarisse, qui voyait les Américains comme étant «de la même race que les Français» (106), c'est-à-dire des maîtres du monde qui ne font que «chier sur le reste de l'univers» (106) et sont la cause de l'état catastrophique du pays, ce à quoi Mika 
répondait: «Ces discours de victimisation, tu le sais aussi bien que moi, ne servent pas notre cause, mais renforcent une irresponsabilité collective» (107). Malgré son amertume face à ses agresseurs, et face à tous ceux qui la jugent et croient qu'elle a elle-même attiré son malheur en s'obstinant à écrire ses articles, Mika ne nourrit pas de sentiment de vengeance.

À la différence de sa grand-mère, Junon - également très affectée par cette nuit du 5 janvier 1958 puisque Soledad a depuis souffert de dépression grave et n'a jamais su être une mère - porte en elle un besoin de vengeance qui n'attend qu'à être expulsé: «J'ai l'impression de vomir mes entrailles, tandis que ma détresse, mon besoin de vengeance demeurent là, au-dedans de moi, comme un enfant terrible dans mon sein, un enfant qui prendra ma vie le jour où il fera entendre sa voix, je le sais. » (206) Parmi les voix de femmes qui s'élèvent contre les injustices, toutes n'ont donc pas la force et la sagesse de Mika pour faire face aux horreurs du duvaliérisme. Le roman soulève ainsi des questions sur le besoin de justice et le désir de vengeance. Junon est venue en Haïti faire un documentaire sur cette nuit qui a changé la vie de sa mère, mais l'aveu de cet «enfant terrible» qu'elle porte en elle dévoile que ce n'est pas seulement le devoir de mémoire qui la motive. La fin est à cet égard révélatrice, d'autant que le dernier chapitre n'est plus raconté par Junon ni par Mika, mais par un narrateur omniscient qui instaure une sorte de distance. Avant de se diriger à l'aéroport pour son retour en Espagne, Junon se rend à la demeure de celui qui a violé et torturé Soledad (et bien d'autres femmes) et aperçoit une jeune fille, accompagnée de deux hommes, sur les lieux. Au bourreau de sa mère, c'est-à-dire à son père biologique, nommé Astrel Benjamin, cette jeune fille déclare: «Qui vous parle de justice? Avons-nous prononcé ce mot?» (217) La colère, la rage, voire le désir de vengeance des enfants issus des crimes horribles de ce personnage, sont tout à fait compréhensibles, mais le dénouement laisse entendre que l'âme tourmentée de Junon ne peut trouver la paix que dans la mort de cet homme, brûlé vif par la jeune fille et ses acolytes.

La narratrice est consciente que répondre à l'appel de la voix qu'elle porte dans son sein comme un enfant indésirable représenterait un point de non-retour. De manière intéressante, Junon arrive à assouvir son désir de vengeance sans avoir à agir selon cette voix. Au moment de quitter la demeure de Benjamin, elle pressent ce qui va se produire, mais elle part tout de même, sans aucune tentative pour changer le cours des événements. Ce qui devait arriver arrive, et les tout derniers mots du roman offrent une scène cinématographique saisissante:

Lorsque l'auto de Junon parvint tout au haut du morne La Vallée, là où elle devait prendre l'embranchement vers la route menant à la frontière, elle s'arrêta. Tout en bas, elle vit s'élever de gros nuages de fumée et d'immenses langues rougeâtres. [...] Là-bas, tout en bas, le feu crépitait. Telle une couleuvre avide, il encerclait la maison. Un jour comme aujourd'hui, clama Junon, un jour, même avec des cheveux blancs, je reviendrai danser et pisser sur ces cendres. À présent, se dit-elle, en s'engageant dans l'embranchement, je vais simplement m'asseoir et regarder s'éteindre tout ce qui brûlait en moi. (220) 
Dès lors, ce n'est pas son documentaire qui permet à Junon d'éteindre le feu de la vengeance en elle, mais le meurtre commis par d'autres. Subsiste néanmoins, en dépit du soulagement et de la satisfaction, une étincelle de rancœur, comme le souligne la promesse de revenir pisser sur les cendres de la maison du mort.

Les émotions des personnages de Femmes au temps des carnassiers sont exprimées avec force et détails. Fidèle à son engagement littéraire de faire entendre la voix des victimes, Marie-Célie Agnant poursuit, avec ce livre, une œuvre qui dénonce les blessures et les injustices de l'histoire. Aux violeurs et aux tueurs, l'écrivaine oppose ici des «femme[s] debout» (84), des "guerrière[s]» (83) qui peuplent son imaginaire. Le roman prend soin de ne pas faire de Junon une "carnassière», car si on sent bien son envie de tuer ou de torturer Astrel Benjamin, elle ne l'a pas fait ou, pour le dire autrement, elle n'a pas eu à le faire. Le dénouement permet effectivement à Junon de vivre sa revanche avec bonne conscience et répond peut-être également aux attentes des lecteurs. Il y a toutefois quelque chose de troublant dans cette fin, car ne propose-t-elle pas le glissement du devoir de mémoire vers le devoir de vengeance ou, à tout le moins, l'entrelacement des deux? Un peu avant la scène finale, et avant même que Junon ne surprenne la jeune fille et ses complices, la narration révélait déjà le croisement des deux projets: «Un nuage épais, fait de rage puissante, lui enveloppait le cœur. Elle [Junon] était prête à affronter l'enfer, se disait-elle, prête à tout pour donner une voix à cette histoire. L'heure des comptes avait sonné.» (212-213)

Personne ne peut rester indifférent à la douleur de Junon et à celle de tous les enfants nés du viol des "carnassiers», et on ne peut qu'imaginer leurs sentiments de frustration et de désespoir devant l'impunité des criminels. Cependant, si le roman est un «lieu de mémoire» dans la mesure où «une histoire tue est une histoire tuée», comment interpréter une mémoire qui suggère que la justice, ou la libération, se trouve dans la vengeance? Car s'il n'excuse pas le meurtre, le récit de Junon tend à l'expliquer, voire à le justifier. Peut-on, en ce sens, parler de Junon comme d'une «femme debout» au même titre que Mika? Les différentes postures des personnages soulèvent plusieurs questions, mais c'est aussi là que réside l'intérêt du roman, qui nous force à nous mettre dans la peau de toutes les victimes de cette nuit du 5 janvier 1958.

Dans Journal d'un écrivain en pyjama, Dany Laferrière revendique l'appellation «roman", comme il le fait pour tous ses livres, même pour ceux dans lesquels il n'y a ni aventures ni autres personnages que lui-même et son narrateur ${ }^{4}$. Selon l'auteur, c'est la liberté même de ce genre qui lui permet de qualifier son œuvre d'ensemble romanesque:

Je sais qu'il y a des écrivains qui n'arrivent même pas à relire leur livre après publication. Presque un rejet. À la sortie d'un livre, je passe un mois à le relire, comme

4 Dany Laferrière, Journal d'un écrivain en pyjama, Montréal, Mémoire d'encrier, coll. «Chronique», 2013, p. $44-45$. 
si je n'en étais pas l'auteur. Puis, un jour c'est fini. Je ne le reprendrai pas avant dix ans. Pour le réécrire. Je ne m'embarrasse alors pas de scrupule. J'ajoute des choses, j'en élimine d'autres, je touche au style. Je me sens totalement libre. Est-ce pourquoi j'affirme que tous mes livres sont des romans? Même ceux qui ressemblent à des essais critiques ${ }^{5}$.

Tout ce qu'on ne te dira pas, Mongo ${ }^{6}$ fait partie des livres qui « ressemblent à des essais critiques», en plus d'être, à plusieurs égards, une réécriture d'autres «romans». Mélangeant fiction, dialogues, réflexions, opinions personnelles et chroniques à la radio, l'ouvrage est présenté en quatrième de couverture comme une "conversation [qui] débute comme dans un roman de Diderot» et une «longue lettre d'amour au Québec».

Fraîchement arrivé à Montréal, Mongo est un Africain qui s'est lui-même donné son prénom en hommage à l'écrivain camerounais Mongo Beti, un homme de «colère» qui «ne pren[ait] [sic] rien pour acquis» (8) et "cuvait en lui une haine folle pour toute forme d'injustice» (115). Le jeune homme désorienté et voué à un «destin d'écrivain» (158) rappelle au narrateur son propre parcours, lui qui a atterri à Montréal en 1976, après avoir fui la dictature de Baby Doc et possiblement la mort. Beaucoup de temps s'est écoulé depuis les premières années, comme on peut en juger par le travail que fait désormais l'auteur devenu célèbre:

- Et que faites-vous pour vivre? [lui demande Mongo.]

- Je parle à la radio le matin.

- De quoi?

- Je raconte ce qui me passe par la tête.

- Et on vous paie pour ça?

- Moins vous travaillez, mieux on vous paie. Je travaille dix fois moins qu'à l'usine,

et je suis payé dix fois plus. (9)

La notoriété ne change pourtant pas tout, comme en témoigne la question d'une jeune femme, lors d'une émission de télévision à Paris, qui lui demande pourquoi il n'est pas en Haïti au lieu d'être en France, puisque c'est «son pays»:

Si je rencontre un Français à New York, je ne vais pas lui demander pourquoi il n'est pas en France. Il faudrait que je sois un ami intime. Dès qu'on vient d'un pays pauvre, on se fait apostropher par des gens que l'on ne connaît pas. C'est une façon de vous dire que vous pouvez bien être un écrivain célèbre, je peux à tout moment vous rappeler vos origines. (74)

Alors que cette Française lui demande pourquoi il est en France, les Québécois se demandent, quant à eux, pourquoi il envisage de s'installer définitivement à Paris. Ce qui est particulièrement intéressant dans cette «longue lettre d'amour au

5 Ibid., p. 254.

6 Dany Laferrière, Tout ce qu'on ne te dira pas, Mongo, Montréal, Mémoire d'encrier, coll. «Chronique», 2015, $304 \mathrm{p}$. 
Québec ${ }^{7}$ », c'est que le narrateur y révèle en fait l'amour étouffant des Québécois. Comme dans une relation de longue date, après le coup de foudre initial, on avait vu Laferrière partir sans faire d'histoire parce que, même s'il est resté à Miami pendant douze ans, il revenait souvent, de sorte qu'on s'était à peine aperçu qu'il était parti. Or, depuis son élection à l'Académie française, on voudrait à tout prix le retenir ici, le garder pour nous:

Mes déplacements sont surveillés et je ne sais pas toujours comment prendre cela. Sur un plan personnel ou sur un plan collectif. S'agit-il d'un moi qui aurait suscité un sentiment si fort chez les gens qu'ils répugnent à me savoir ailleurs? Ou veut-on me rappeler que je ne dois pas quitter un pays qui m’a protégé durant toutes ces années? [...] Malgré tout, vous savez comment c'est, l'amour, il peut être étouffant, alors je ressens un point chaud et froid au cœur chaque fois qu'on me questionne sur l'endroit de ma résidence. Mine de rien, depuis un an, toute conversation se termine par : tu ne vas pas rester là-bas? Là-bas, c'est Paris, comme là-bas fut Montréal pour ma mère. [...] Chaque interrogation (tu vas partir là-bas?) me dit qu'on tient à moi en même temps qu'on doute de moi. (133-134)

Ce doute est aussi lié à l'habitude du narrateur d'étaler une pléthore de renvois à des écrivains étrangers dans son œuvre et dans ses entretiens, tandis que les auteurs québécois y sont, à quelques exceptions près, absents: "Si vous êtes un intellectuel, pourquoi toujours citer les écrivains d'ailleurs quand vous êtes ici? Comme partout, on aime ici ceux qui se donnent la peine de s'intéresser à la culture du pays. C'est la moindre des choses.» (212) En conséquence, et quoique Borges soit toujours très présent, le narrateur parsème son dernier livre de références québécoises. On rencontre donc au passage Gaston Miron (dont un extrait du poème «Pour mon rapatriement» est cité en exergue), Réjean Ducharme, Jacques Ferron, Germaine Guèvremont, Claude Gauvreau, Émile Nelligan, Anne Hébert, Monique Proulx, etc. Est-ce une façon d'exprimer sa gratitude? Car ce que devra retenir Mongo, c'est que le Québec, qui a conservé son âme judéo-chrétienne en dépit de la Révolution tranquille, a un cœur charitable mais s'attend à une reconnaissance. Cette idée met Mongo en colère, et encore plus son oncle, mais le narrateur croit au contraire que l'immigrant devrait «prendre la peine en arrivant de dire merci aux gens qui l'ont si chaleureusement accueilli, avant de s'incliner devant la loi» (151).

Mongo découvrira d'autres choses que «tout le monde sait» (88) et qu'on ne lui dira pas. Par exemple, qu'il ne peut "pas arriver comme ça» (88) et s'imposer

7 C'est d'ailleurs ainsi que la critique a lu Tout ce qu'on ne te dira pas, Mongo. Pour ne mentionner que quelques exemples, citons Danielle Laurin du Devoir: «Si le livre semble s'adresser d'abord à Mongo, puis au nouvel immigré au sens large, il se veut aussi un miroir offert aux Québécois, à ceux que Dany désigne comme les natifs. C'est fait avec humour, et beaucoup d'amour.» ("Conseils d'un Immortel à un nouvel immigré», Le Devoir, 14 novembre 2015). Marie-Louise Arsenault, à l'émission «Plus on est de fous, plus on lit!», a de son côté dit du livre de «l'écrivain superstar», qu'elle recevait "très fièrement», qu'il était «plein de tendresse pour le territoire [québécois]», que l'auteur a "choisi» (émission du 11 novembre 2015). Pour sa part, Chantal Guy reprend tout simplement les propos de Laferrière dans le titre de son article: «Tout ce qu'on ne te dira pas, Mongo : une lettre d'amour au Québec» (La Presse, 8 novembre 2015). 
aux gens en s'assoyant à la table d'un café sans y être invité; que le sourire n'est pas «une invitation, ici» (77), mais «une mimique du visage qui sert à tenir les gens à distance» (165); qu'il devra apprendre à s'excuser parce qu'«[o]n y accorde une grande importance ici» (137); que, s'il est «invité à un souper» (210), il devra apporter «un plat de son choix. Et une bouteille de vin» (210); qu'il faut «éviter, ici, de même frôler la personne avec qui l'on converse» (78), car «le Québec est un pays de grands espaces» (78), si bien qu' "[à] trois mètres de distance, vous piétinez déjà l'espace du voisin. À un mètre, c'est une agression. Et à cinquante centimètres, on crie au viol» (165). On ne dira pas non plus à Mongo que la Grande Noirceur n'est pas « cette glaciale nuit qui part de la fin décembre jusqu'au début avril» (167); qu'il ne faut pas aborder le sujet «trop explosif» (230), «tabou» (231), des Amérindiens dans les soirées mondaines; et qu'ici, «[t]out est excusable, sauf la vanité, qui se définit par le fait de se prendre pour un autre» (267). Et, pour ne pas donner l'impression de se prendre pour un autre, il faut vite tutoyer les gens, car «[n] ous sommes tous au même niveau, jusqu'à ce que tu découvres que c'est illusoire» (46).

Pour aider Mongo et tous les nouveaux venus (évidemment francophones), le narrateur propose également un petit lexique dans lequel on retrouve la définition de quelques mots-clés, dont celle du Québécois : «Un Québécois : C'est un individu prêt à mourir pour une langue qu'il ne cherche pas à bien écrire» (162). Plus loin, en anticipant les reproches, il note qu'avec les nouvelles générations, « on parle plus d'environnement à protéger que de territoire à défendre. Il ne reste plus que la langue, et on parle d'elle plutôt que de chercher à bien la parler. Oh là, bien parler, ce n'est pas parler bien!» (244) Si le stéréotype du Québécois défendant corps et âme une langue qu'il ne maîtrise pas est reconduit - et entre autres expliqué par le legs du père qui, après s'être muré dans le silence, s'est réfugié dans la taverne où, trop ivre, il oubliait le nom de sa femme et se mettait à l'appeler «chose» (43; en italique dans le texte) - , les clichés «à oublier» sont: «Le Français est arrogant. L'Anglais, dominateur. Le Québécois, humble et travailleur.» (162) Qui aime bien châtie bien? Après la définition du Québécois, le narrateur donne celle de l'«immigré» : «Un immigré: Tout individu arrivé ici après la mort de Maurice Le Noblet Duplessis [...]. Avant, on disait un survenant.» (163) A-t-on vraiment déjà dit «immigré» au Québec? L'écrivain lui-même a déjà employé le terme «immigrant», mais voilà qu'il est devenu interdit: «Comme je déteste ce mot si laid d'immigrant - qui n'est même pas français. On dit immigré» (247). "Immigrant» est mis en italique, comme «blonde» (20), "chanteur de pomme» (21), «niaiseux» (41), «taiseux» (41), «tomber en amour» (177), «un cassé» (223) et «la patente» (276). Le mot est pourtant dans les dictionnaires - pas seulement québécois et anglais - et figure même dans le Dictionnaire de l'Académie française, qui exclut par ailleurs les «aberrations» que sont "professeure», «écrivaine» et "auteure», qu'on utilise sciemment au Québec. Est-ce pour mieux s'en prendre (encore une fois) à la littérature migrante que le narrateur propose cette leçon de vocabulaire, comme si «immigrant» ne servait qu'à désigner quelques auteurs connus? "J'ai l'impression d'avoir une étoile au front chaque fois qu'on dit de moi que je suis un écrivain immigrant, confie-t-il.» (247) Vraiment?

Dans ce livre éclectique, Laferrière donne aussi la parole à l'Empire du Milieu qui s'adresse à l'Occident dans sa «Lettre de la Chine»: «Nous vous avons 
fait apprécier le buffet chinois dont le menu peut comprendre 92 plats qui se ressemblent, sans être tout à fait pareils, un peu à notre image. C'est notre arme de destruction massive. Elle s'attaque à vos corps que nous rendons obèses pendant que nous restons minces.» (113-114) Tout ce qu'on ne te dira pas, Mongo me fait justement penser à un buffet chinois. À l'instar de plusieurs autres livres de l'auteur, ce dernier contient des opinions sur une multitude de sujets, ce qui permet aux lecteurs de l'ouvrir au hasard et de choisir les «plats» qui les intéressent. Il serait même possible d'entamer les passages de la fin avant ceux du début, sans vraiment être dérouté, comme quelqu'un qui, au buffet, commencerait par le très chinois millefeuille plutôt que par le egg roll, sans faire sourciller les serveurs. Et, comme dans un buffet, on peut trouver deux fois le même plat, placé à chacune des extrémités de la salle et faire croire aux clients qu'il y a une grande variété de choix. L'écriture n'est pas une "arme de destruction massive», mais une "arme de séduction massive». Ce n'est pas pour rien que, dans sa «Réponse» au «Discours de réception» de Laferrière, l'Académicien franco-libanais Amin Maalouf a déclaré: «Vous n'êtes pas dans le militantisme, mais dans la séduction ${ }^{8}$.» Pour nous mettre l'eau à la bouche, le narrateur présente d'abord un personnage exotique, Mongo, et évoque ensuite ses relations sexuelles avec Catherine, une jeune Québécoise qui décide de faire son mémoire de maîtrise sur son amant africain, en «l'observant», comme on le ferait d'un objet d'art ou d'un petit animal dans une cage. Ce qui ne veut pas dire qu'elle ne soit pas amoureuse de Mongo. La relation "explosive», selon l'adjectif fréquemment utilisé par l'écrivain pour décrire le rapport entre le Noir et la Blanche, encourage donc les lecteurs à dévorer le livre. Conscient, toutefois, que le repas est lourd (il y est question de sexe et d'exotisme, mais surtout de langue, d'immigration, de religion, d'indépendance, de fédéralisme, de dictature, d'exil, de racisme, de discrimination positive, de féminisme, d'accommodements raisonnables, etc.), le narrateur prend soin de glisser, entre quelques bouchées, de bonnes tasses d'humour pour nous aider à digérer le tout. L'humour de Laferrière, accessible, se situant habilement (la plupart du temps) à la lisière du "politiquement correct», est en partie ce qui nous fait oublier que, s'il nous donne souvent quelque chose à nous mettre sous la dent, il a tendance à nous servir les mêmes recettes. Le narrateur-écrivain admet d'ailleurs que la rencontre imaginaire avec Mongo n'est qu'un prétexte pour remonter le temps et revenir sur son parcours: «Et si je suis revenu sur mes pas, c'est pour te croiser en chemin. Ainsi le lecteur aura parfois l'impression de lire des passages entiers qui lui sembleront familiers.» (158)

Malgré l'impression de «déjà lu» ou de "déjà entendu à la radio », il est impossible de trouver un livre de Dany Laferrière complètement inintéressant. Il y aura toujours une phrase pour nous faire rire, un point de vue pour nous pousser à réfléchir, une idée brillante pour nous éclairer. En fermant Tout ce qu'on ne te dira pas, Mongo, cette «longue lettre d'amour» aigre-douce, je me suis tout de même demandé pourquoi on aimait tant le buffet chinois au Québec, alors qu'on y offre presque invariablement les mêmes plats, relevés, ici et là, il est vrai, d'une nouvelle sauce.

8 Dany Laferrière, Dany Laferrière à l'Académie française. Discours de réception. Réponse d'Amin Maalouf, Montréal, Boréal, 2015, p. 79. 